\title{
Active Noise Control with a Simplified Multichannel Genetic Algorithm
}

\author{
Antonio Minguez ${ }^{\dagger}$ \\ Dpto. Ingenieria Audiovisual y Comunicaciones (DIAC), Escuela Universitaria de Ingenieria Tecnica de \\ Telecomunicaciones (EUITT), Universidad Politecnica de Madrid (UPM), Ctra. Valencia Km.7, 28031 Madrid, Spain
}

\begin{abstract}
Manuel Recuero ${ }^{\dagger}$
Instituto de Investigacion del Automo vil (INSIA), Universidad Polit9 cnica de Madrid (UPM), Ctra. Valencia Km.7, 28031 Madrid, Spain
\end{abstract}

(Received 5 September 1999; accepted 16 November 1999)

\begin{abstract}
A multichannel genetic adaptive algorithm has been developed for active noise control applications. This algorithm is based on the classical genetic algorithms. The FIR/IIR filter coefficients are searched for in a random way, following the biological evolution stages: reproduction, mutation and selection. In an active noise control configuration these algorithms do not require the cancellation path estimations between the control speakers and the error microphones, so there is a huge complexity reduction compared with filtered-X LMS algorithms, which make on-line estimates. Besides, they do not present the local minima problem of the conventional gradient descent-type algorithms (such as filtered-U LMS). However, genetic algorithms show some disadvantages: slow convergence and high residual error noise. The algorithm presented overcomes these disadvantages and reduces the computational complexity removing the reproduction stage. The slow convergence and the final residual are improved by controlling the mutation parameter, generation after generation, and with small chromosome populations. Good results have been obtained for periodic signals, with attenuations between 15 and 20 $\mathrm{dB}$. It has also been tested in a real active noise control application for reducing the automobile engine noise.
\end{abstract}

${ }^{\dagger}$ Member of the International Institute of Acoustics and Vibration (IIAV)

\section{INTRODUCTION}

Multichannel active noise control is used when an acoustic noise reduction is going to be made in a noisy enclosure (global space acoustic attenuation). Several control acoustic sources are located inside the enclosure in order to produce an opposite acoustic noise field in some points, where the "error" microphones are placed. A reference signal is measured, by means of an electroacoustic transducer, and supplied to a controller in order modify the amplitude and phase of the signal and produce the output control signals, that are fed to the multichannel power amplifier and then to the speakers (acoustic control sources).

In a monochannel active noise controller an "ideal attenuation" can be obtained at the error microphone point, modifying the amplitude and the phase of the reference signal. The block diagram of the system is shown in Fig. 1. The controller chosen was an adaptive digital filter which is used to follow in real time any amplitude and phase variation, and optimise the acoustic attenuation.

The transfer function of the controller can be obtained in the $Z$-domain from the system model. $H_{e}(z)$ is the global transfer function from the output control signal to the input error signal, and consists of the following cascade transfer functions: digital-analogue converter, power amplifier, speaker actuator to produce acoustic waves, acoustic path between the speaker and the microphone, microphone transducer, signal amplifier and the analogue-digital converter. $R(z)$ represents the transfer function that links the noise source, $N(z)$, with the digital input reference signal, that is, the transducer transfer function plus the analogue-digital converter. $P(z)$ is the transfer function that links the noise source and the digital error input signal: acoustic path to the microphone, microphone transducer, signal amplifier and the analogue-digital converter. The transfer function for the controller that makes $E(z)=0$ is given by Eq. (1)

$$
C(z)=\frac{-P(z)}{R(z) H_{e}(z)} .
$$

An adaptive FIR (finite impulse response) filter can be used to predict the output control signal for periodic noise signals but a causality condition must exist for random noise signals, that is, the delay introduced by $R(z)$ plus the $H_{e}(z)$ delay must be shorter than the $P(z)$ delay. If the transducer that produces the reference signal is a microphone, acoustic feedback can occur and the optimal function for $C(z)$ is now given by Eq. (2)

$$
C(z)=\frac{\frac{-P(z)}{R(z) H_{e}(z)}}{1-F(z) \frac{P(z)}{R(z) H_{e}(z)}},
$$

where $F(z)$ is the transfer function, in the $Z$-domain, between the output control signal and the reference signal. The acoustic feedback introduces poles in the response of the model that must be removed by an adaptive IIR (infinite impulse response) filter.

In a multichannel active noise control system, as shown in Fig. 2, there are $M \times K$ acoustic paths - included in $H_{\text {eii }}(z)$

pp 27-32) 